\title{
Visualization of paper structure by optical coherence tomography: Monte Carlo simulations and experimental study
}

\author{
M. Yu. Kirillin \\ mkirillin@yandex.ru
}

\section{E. Alarousu}

\section{T. Fabritius}

\section{R. Myllylä}

\section{A. V. Priezzhev}

\begin{abstract}
University of Oulu, Optoelectronics and Measurement Techniques Laboratory, P.O. Box 4500, 90014, Oulu, Finland

M.V. Lomonosov Moscow State University, Physics Department, Vorobiovy Gory, Moscow, 119992, Russia

University of Oulu, Optoelectronics and Measurement Techniques Laboratory, P.O. Box 4500, 90014, Oulu, Finland

University of Oulu, Optoelectronics and Measurement Techniques Laboratory, P.O. Box 4500, 90014, Oulu, Finland

University of Oulu, Optoelectronics and Measurement Techniques Laboratory, P.O. Box 4500, 90014, Oulu, Finland

M.V. Lomonosov Moscow State University, Physics Department, Vorobiovy Gory, Moscow, 119992, Russia
\end{abstract}

This paper analyzes the capability of an optical coherence tomography (OCT) setup to visualize paper structure by comparing experimental and Monte Carlo (MC) simulated OCT images. In the experiments, we used an OCT setup at $910 \mathrm{~nm}$ with the coherence length of an SLD source of $7.5 \mu \mathrm{m}$. Several different refractive index matching agents were applied to paper samples to enhance the visualization of their inner structure. The obtained OCT images show improved contrast in inner structure and enhanced visualization depth. A set of corresponding simulations were then performed using a previously developed MC code. A multilayer paper sheet model with non-planar fiber-air interfaces was developed to characterize the paper samples. MC simulations were carried out to study the effect of the setup parameters on the obtained OCT images. A comparison with the experimental data shows that the developed model provides a satisfactory correspondence between simulated and experimental images. [D0I: 10.2971/je0s.2007.07031]

Keywords: OCT, Monte Carlo simulations, multiple scattering, paper, optical clearing

\section{Introduction}

Each paper sample has a unique structure which can be represented by a particular stochastic network of cellulose fibers [1]. Changing the properties of paper is usually performed by adding fillers of various sizes to paper pulp during production [2]. Also paper samples contain fines, which are small parts of fibers. The thickness of forming cellulose fibers typically varies from 10 to $40 \mu \mathrm{m}$ (fiber diameters in different types of wood are: in birch $22 \mu \mathrm{m}$, in eucalyptus $16 \mu \mathrm{m}$, in pine $20-$ $35 \mu \mathrm{m}$, in spruce 19-33 $\mu \mathrm{m}$ [3]), while their length can reach several millimeters. Paper samples usually vary in thickness from 50 to $150 \mu \mathrm{m}$. An important quality control aspect in paper production is the monitoring of paper structure with an appropriate imaging technique.

In paper production the techniques able to provide real time paper monitoring are required. This is the key requirement to the technique. The properties of paper to be controlled among others are paper thickness and porosity.

Optical coherence tomography (OCT) is an elaborate method for non-invasive imaging of the inner structure of optically non-uniform objects [4]. This fact allows us to suppose that OCT could be an effective tool for the paper industry, where fast and precise techniques are necessary for paper property control during production. OCT provides a high scanning rate, high resolution and does not necessitate long preprocessing of the samples $[5,6]$. On the other hand, current methods are scanning electron microscopy (SEM) and laser confocal microscopy (LCM), both of them provide higher resolution compared to OCT. However, the former requires additional time-consuming processing of the studied object before imaging which includes filling the air pores with special agent and further precise cutting of the sample for obtaining the crosssection for imaging. The latter has a limited imaging depth (few tens of micrometers for paper, which is smaller than provided by OCT) restricted by multiple scattering in the sample under study. Due to this fact the imaging of in-depth paper cross-section by LCM can also be obtained only after cutting the sample. Usually LCM is utilized for studying the paper surface profile. More than that, SEM and LCM techniques provide the scanning times longer than OCT (in modern OCT systems, especially the Fourier domain ones, the A scans can be obtained at kilohertz rate).

Usually, in the quantitative characterization of a paper sample's optical properties, paper is considered as a uniform scat- 
tering medium in frames of the Kubelka-Munk theory [2]. However, this approach does not account for the complex structure of paper and seems hardly applicable to the analysis of OCT images of paper samples [5, 7]. Although the sophisticated 3D paper model, published by Green et al., describes the structural features of paper very well [8], their model is complicated and requires a long calculation time, making it hardly practical for the simulation of OCT images. A simpler model which adequately describes the structural properties of paper would be more practical for implementation.

From the microscopic point of view, light scattering is caused by a distortion of the electromagnetic field in an area with a variable refractive index; from the macroscopic point of view, it is caused by the reflection and refraction of light at the boundaries of media with different refractive indexes [9]. A scatterer can be considered as an area whose refractive index differs from that of the environment. If the refractive index mismatch $n_{1} / n_{2}$ is rather small and the scatterer size $\mathrm{R}$ is quite large compared to the wavelength $\left(n_{1} / n_{2}<1.05, R \gg \lambda\right)$, the anisotropy of scattering is large and the average change in the direction of photon propagation after the scattering event is insignificant. In the opposite case, the effect of scattering is fairly high, which leads to the chaotization in the direction of photon propagation and, consequently, to blurring in the OCT image. To reduce the effects of light multiple scattering on OCT images, refractive index matching agents (RIMAs) [10] are usually applied to produce an optical clearing effect. This effect is based on the substitution of initial medium with a significantly different refractive index by a medium with refractive index value close to that of the scattering components. This substitution results in changing the scattering properties of the object under study $[10,11]$. A potential RIMA for a paper sample should satisfy the following requirements: (i) its refractive index should be close to that of the cellulose fibers $\left(n_{f}=1.53\right)$ forming the basis of the sample; (ii) it should not penetrate into the fibers, filling only the air gaps inside the sample: (iii) it should not react chemically with the fibers, fillers or other components of the sample.

In our previous work [5], we have analyzed different RIMAs to find which ones best satisfy the above-listed requirements. It was shown that 1-pentanol and benzyl alcohol are advantageous for the optical clearing of paper. Further studies then established that isopropanol is also applicable for the purpose. In this work, we analyze how these three RIMAs affect the OCT imaging of paper samples both experimentally and by a numerical simulation implementing a previously developed Monte Carlo code. To avoid additional scattering effects caused by fillers, special paper samples without fillers were prepared to facilitate the interpretation of the experimental images and to provide a basis for comparison, even though the proposed model can be extended to account for the presence of fillers. Another aim involved studying the effect of such OCT system parameters as the detector's numerical aperture and the light source's coherence length, on the obtained images. These parameters are usually fixed for a particular OCT system, and a rebuild of the setup is needed to change them. Monte Carlo simulations, however, allow varying these parameters quite freely.

\section{MATERIALS AND METHODS}

\subsection{Experiment}

This study used a specially prepared paper sample lacking any fillers. Its thickness was about $150 \mu \mathrm{m}$ and porosity about $40 \%$. As already mentioned, three RIMAs, whose refractive indices are shown in Table 1, were used for the optical clearing of the samples.

\begin{tabular}{|c|c|}
\hline RIMA & $n$ \\
\hline Isopropanol & 1.375 \\
\hline 1-pentanol & 1.407 \\
\hline Benzyl alcohol & 1.538 \\
\hline
\end{tabular}

TABLE 1 Refractive indices of the refractive index matching agents(RIMA)

Experimental images of the samples were obtained with a commercially available fiber-based OCT device produced by the Institute of Applied Physics of RAS (Nizhny Novgorod, Russia). This setup uses a superluminescent diode having a center wavelength of $910 \mathrm{~nm}$ and a FWHM spectral width of $49 \mathrm{~nm}$ as a light source and has coherence length of 7.5 $\mu \mathrm{m}$, transversal resolution of $9 \mu \mathrm{m}$, and the detection angle of its measuring head is $6^{0}$ in air. The irradiated power to the sample is $0.6 \mathrm{~mW}$. The signal is modulated to $1.415 \mathrm{MHz}$ Doppler frequency and then after detection amplified, filtered, demodulated, A/D-converted and saved to the computer via USB channel. A scanner inside the OCT-measurement head gives an adjustable scanning range between 0 and $2.2 \mathrm{~mm}$ along the $x$ - and $y$-axes which can be extended by interchangeable objective at the expense of the transversal resolution. A schematic diagram of the experimental arrangement is given in Figure 1. The setup is capable of producing 2D-

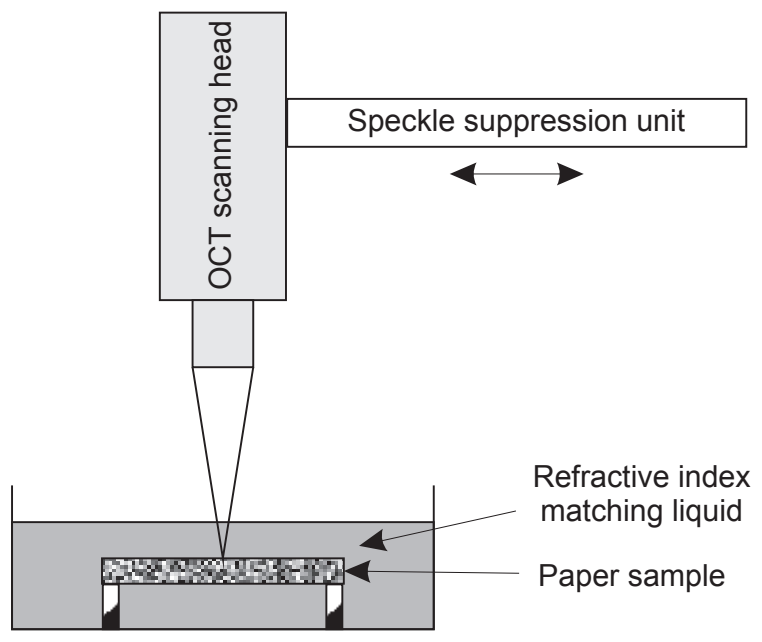

FIG. 1 Schematic of the experimental arrangement

and 3D-OCT reconstructions of the samples within few seconds. A 200x200 pixels 2D-slice image without averaging can be recorded in 1.9 seconds. 
The sample to be measured was placed into a cuvette with a circular holder beneath the paper sample. After placing the sample into the cuvette, the latter was filled with a refractive index matching liquid so that the sample was completely immersed in the liquid and, thus, totally soaked. To decrease the effect of speckles, a piezo element with a frequency rate of about $15 \mathrm{~Hz}$ was connected to the scanning head for the spatial averaging of A-scans along the scanning direction. Each sample was imaged with and without speckle suppression. A new sample was used for each RIMA, because it is impossible to restore the initial structure of the sample after the application of a RIMA.

\subsection{Monte Carlo simulations}

As already mentioned above, paper has a fairly complicated structure, which makes the analysis of light propagation in it quite sophisticated. In an earlier publication [7], we have developed a multilayer model of paper comprising fiber and air (fiber-free) layers for the simulation of OCT images. On one hand, this model is more complicated than the uniform medium model used for the evaluation of paper optical properties within the framework of the Kubelka-Munk [2] or diffusion theories [12]-[14]. On the other hand, it is simpler than the fiber network model developed in Ref. [8]. As a result, it enables faster calculations, while accounting for the nonuniform structure of paper. Unlike the Kubelka-Munk and diffusion theory, this model can take into account the porosity of the sample as well as filler and fine particles [7]. An alternative model, treating paper as a porous medium with ellipsoidal pores, was proposed in Ref. [15].

Monte Carlo simulations of OCT images were performed by a consecutive step-wise calculation of individual in-depth scans (A-scans) along the z-axis, followed by a further compilation to the matrix of the bitmap object image. The simulated OCT A-scans are represented in a logarithmic scale in accordance with the way of obtaining of the OCT image in the experiment. To obtain one simulated OCT image measuring 1000 by $250 \mu \mathrm{m}$ area $100 \mathrm{~A}$-scans with $10 \mu \mathrm{m}$ steps are required. Considering that only a small portion of all injected photons (circa $3 \%$ ) contribute to the OCT signal and form the image, initially a total of $0.5 \mathrm{mln}$ weighted input photons were used to obtain one A-scan. In this case the calculation time for a single image on a PC running an Intel Pentium processor at $2.5 \mathrm{GHz}$ was about one hour. However, for small numerical apertures $(N A=0.12 \ldots 0.13)$ of the detector, this number of input photons was proved insufficient to provide good image quality. As a result, $5 \mathrm{mln}$ photons were used to obtain one A-scan, resulting in a calculation time of about 10 hours.

In the available literature, we could not find values for the scattering and absorption coefficients of cellulose fibers. Hence, in accordance with ref. [5] and basing also on the data available from $[2,8,16]$, we used the following parameters, obtained from the Kubelka-Munk theory [2], for the average fiber density of $800 \mathrm{~g} / \mathrm{dm}^{3}: \mu_{s}=16 \mathrm{~mm}^{-1}, \mu_{a}=0.5 \mathrm{~mm}^{-1}$, $g=0.94, n=1.53$. The role of the scattering and absorption coefficients is in characterizing the scattering properties of the fibers; the fiber layers are considered to be uniform. We used the empirical Henyey-Greenstein function as phase function, characterizing scattering inside fiber layers, which our previous study found to provide a reasonable approximation [7]. Major scattering events in paper occur at air-fiber and fiber-air boundaries (not inside the fibers), and the shape of the phase function of the fiber layers should not play a significant role while preserving the anisotropy factor value.

Unlike the model presented in Ref. [7], using a paper sample with plain outer boundaries, the model with all non-planar layer boundaries considered in this work looks more realistic. The sample's total thickness was set in accordance with the experimental data. The upper and lower boundaries of the layers were defined by the following analytic expressions:

$$
z(x)=a \sin (2 \pi v x+4 \pi m / 10)+(a / 5) \sin (2 \pi v x+r n d),
$$

$$
z(x)=-a \sin (2 \pi v x+4 \pi m / 10)-(a / 5) \sin (2 \pi v x+r n d),
$$

where $m=1 \ldots 5$ represents the index of the fiber layer, $v$ is the spatial frequency of the boundary sinusoidal shape, chosen equal to $6 \mathrm{~mm}^{-1}, a$ is its amplitude, chosen equal to $2 \mu \mathrm{m}$, $r n d$ is a random value uniformly distributed in the interval $[0,2 \pi]$ and $x$ and $z$ are the transverse and longitudinal coordinates, respectively. Figure 2 presents a cross-section of the model paper sample. The transversal scanning in the simulations is performed along the $\mathrm{x}$-direction.

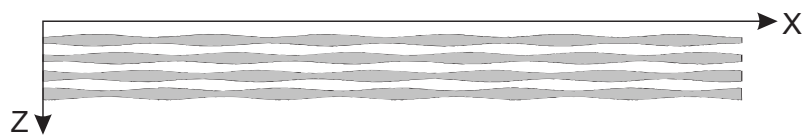

FIG. 2 Cross-section of a model paper sample used in the Monte Carlo simulation of OCT images. The sample comprises 5 fibril layers and 4 air layers. The size of the presented cross-section is 1000 by $140 \mu \mathrm{m}$. A-scans are performed consecutively along the $z$-axis at different $x$ values.

In Eqs. 1 and 2, the random component provides random nonuniformities at the micro-scale to simulate the structural disorder of paper samples. However, due to its scale, this effect is not clearly visible in the figure. More accurate model could be obtained introducing the shapes of the layer boundaries based on real paper geometry, while in this study they are extrapolated analytically for simplification.

When performing simulations we supposed that the applied RIMAs satisfy the requirements mentioned above and do not affect the structure of the sample under study.

\section{RESULTS AND DISCUSSION}

Figure 3 presents experimental OCT images of the studied paper samples obtained with the OCT setup described above. The scale is in $\mu \mathrm{m}$, and optical length is shown at the ordinate axis while physical length is shown at abscissa axes. 
(a)

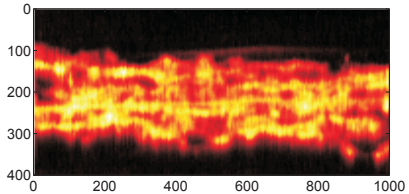

(c)

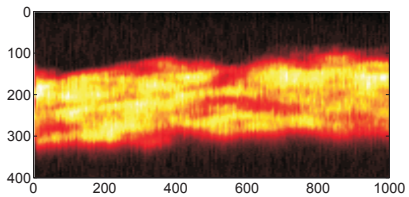

(e)

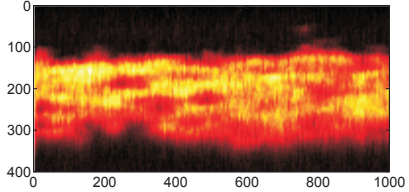

(g)

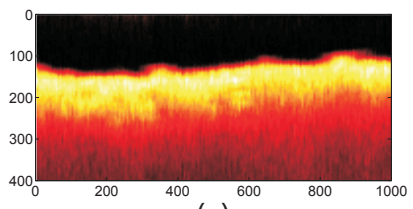

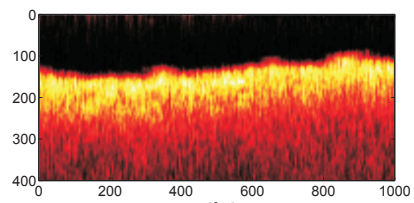

(b)

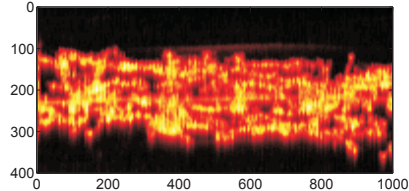

(d)

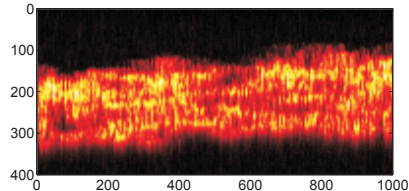

(f)

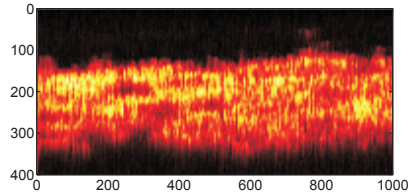

(h)
FIC. 3 Experimental OCT images of the paper samples with speckle suppression (a without RIMA, c - with benzyl alcohol, e - with 1-pentanol, g - with isopropanol) and without it (b - without RIMA, d - with benzyl alcohol, f - with 1-pentanol, h - with isopropanol).

Different pseudo-colors indicate the $z-x$ distributions of the local backscattering efficiency in logarithmic scale (brighter areas correspond to the higher backscattering efficiencies). Comparison of the left-column figures with the right-column ones shows that speckle suppression improves the quality of OCT images in all cases. This proves that the chosen speckle suppression method is effective in the visualization of paper structure by OCT.

The simulated OCT images with and without RIMAs to be compared with the experimental ones (Figure 3) are shown in Figure 4 .

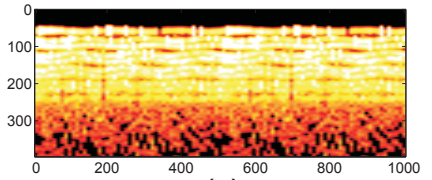

(a)

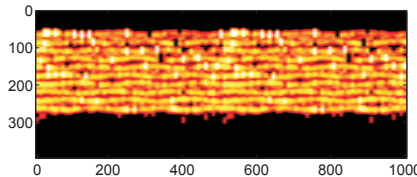

(c)

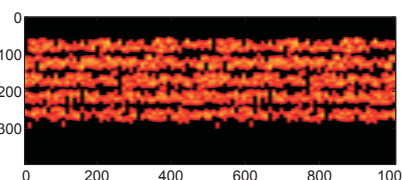

(b)

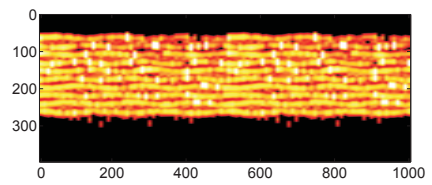

(d)
FIG. 4 Simulated OCT images of the paper samples for $\eta=5^{0}$ and $l_{\text {coh }}=10 \mu \mathrm{m}$ (a without RIMA, b - with benzyl alcohol, c - with 1-pentanol, d - with isopropanol).

This figure shows that simulated images are in qualitative similarity with the experimental ones, however, a more exact coincidence requires the application of more complicated paper models, which are much more time-consuming than the presented multilayer model. On one hand, we are dealing with a complicated task requiring a detailed preliminary examination of the sample for implementing its results to the model; on the other hand, one of the aims of the present study is to propose a simpler paper model, providing reasonable results, for the calculation of light propagation. The proposed multilayer model seems to meet this requirement.

An important criterion for precise non-contact paper thickness measurements is a good image contrast especially in the sample's rear boundary. As the presented images show, the contrast is the lowest when no RIMA is used. In the experimental OCT image, the rear border is not detectable, although it is distinguishable in the simulated image.

We should mention that scattering events occur both at the fiber boundaries and inside the fibers. Multiple scattering in paper samples is contributed by the photons scattered at large angles to initial (forward) direction, which prevents them from leaving the sample into forward hemisphere. Multiple scattering elongates the trajectories of these photons, yielding apparent non-zero OCT signals in the regions corresponding to the depths which exceed the sample's optical thickness. Application of a RIMA significantly decreases the probability of photon scattering at large angles, thereby decreasing the multiple scattering effects. This phenomenon can be observed in the OCT images of samples containing a RIMA. All images are shown in the same color scale to allow a comparison of signal levels in the different cases. Signal intensity is the lowest for benzyl alcohol, because its refractive index is the closest to that of cellulose fibers, resulting in minimal deflections in photon propagation direction at fiber boundaries. However, both the experimental and simulated images demonstrate that the application of RIMA allows visualizing the sample's inner structure. The fact that sample structure is more regular in the simulations than in the real samples allows us to analyze how the inner structure image is formed. Thus, for benzyl alcohol, the signal is formed mostly by photons scattered inside the fibers. In the case of 1-pentanol and isopropanol, showing higher contrast at fiber boundaries, signals are formed by photons reflected from these boundaries.

In this work, we also studied the effects of the coherence length and the numerical aperture $(N A)$ of the detector on the simulated OCT images. As $N A$ depends on the refractive index of the medium and, hence, differs for various RIMAs, we also introduced the detection angle $\eta$, representing the maximal exit angle at which a photon contributes to the OCT signal. We considered the coherence lengths $l_{\text {coh }}$ of 5 and $10 \mu \mathrm{m}$ and detection angles $\eta$ of 5, 10, 15, 20 and 40 degrees.

The effect of coherence length is obvious and is manifested by a decrease in the system axial resolution with a simultaneous increase in signal level, caused by the widening of the coherence function. Figure 5 shows the simulated OCT images of a sample containing benzyl alcohol at two coherence length values: 5 and $10 \mu \mathrm{m}$ and $\eta=15^{0}$.

Figure 6 presents simulated OCT images of paper samples corresponding to $l_{c o h}=5 \mu \mathrm{m}$. The left column shows the ef- 


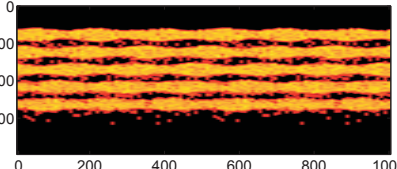

(a)

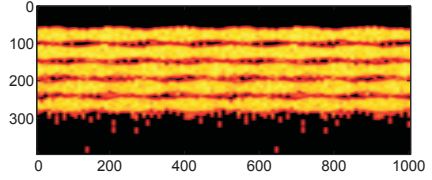

(b)
FIG. 5 Simulated OCT images of a paper sample with benzyl alcohol applied at two values of the coherence length of the light source $\left(\mathrm{a}-l_{\text {coh }}=5 \mu \mathrm{m}, \mathrm{b}-l_{\text {coh }}=10 \mu \mathrm{m}\right)$ for $\eta=15^{0}$.

fects of various detection angle values on the samples with no RIMA applied and the right column shows the corresponding samples containing benzyl alcohol. One can see that the signal levels increase with the detection angle, due to decreasing detector selectivity of single and least scattered photons. However, this decrease in detector selectivity expands the number of multiply scattered photons which contribute to the signal and distort the image. This effect produces an apparent increase in the thickness of the fiber layers and a corresponding apparent decrease in the thickness of the air layers in the obtained images. Besides, as the detection angle increases, contrast in the visualization of the deep layers becomes lower than in the superficial layers, which is clearly observable in images corresponding to $\eta=40^{\circ}$. For smaller detection angle values, the layer contrast remains almost identical.

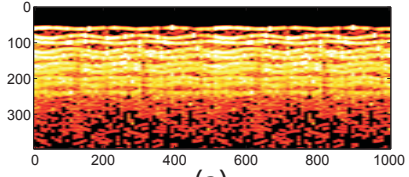

(a)

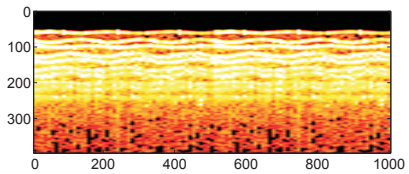

(c)

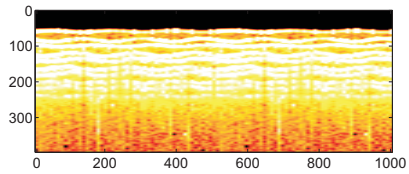

(e)

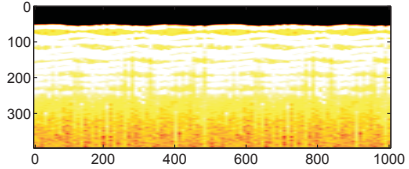

(g)

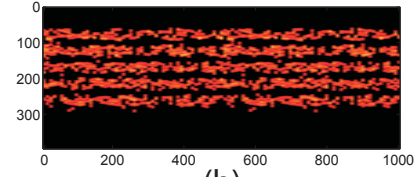

(b)

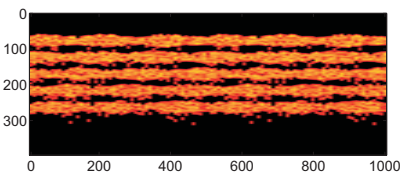

(d)

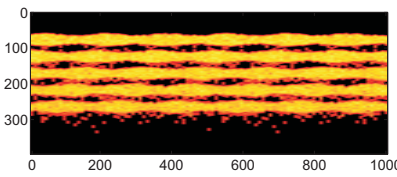

(f)

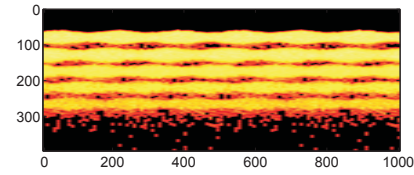

(h)
FIG. 6 Simulated OCT images of paper samples without RIMA applied $\left(\mathrm{a}-\eta=5^{0}, \mathrm{c}\right.$ $\eta=10^{\circ}, \mathrm{e}-\eta=20^{\circ}, \mathrm{g}-\eta=40^{\circ}$,) and samples with benzyl alcohol applied (b $\eta=5^{0}, \mathrm{~d}-\eta=10^{0}, \mathrm{f}-\eta=20^{0}, \mathrm{~h}-\eta=40^{\circ}$, ) at various detection angle values and $l_{\text {coh }}=5 \mu \mathrm{m}$.

As a criterion for the quantitative characterization of the obtained simulated images, we chose the contrast of the rear boundary image. Contrast is defined by the following expression:

$$
K=\frac{I_{b}-I_{a}}{I_{b}+I_{a}}
$$

where $I_{b}$ is the average brightness of pixels corresponding to the rear boundary and $I_{a}$ is the average brightness of pixels corresponding to the air layer. For the samples containing benzyl alcohol, this expression does not work. In the case of almost perfect matching the brightness of the boundary area in the image is the same as the brightness of the fiber layer, while for the case of particular clearing (1-pentanol and isopropanol) the brightness of boundaries is higher that that of the fiber layers. However, when averaging the values on the boundaries are lower than in the middle of the fiber. Due to this fact the value accounted when calculating $K$ for benzyl alcohol is taken as maximum over the fiber layer.

Figure 7 shows the contrast dependence on the detection angle and on the coherence length for various RIMAs. Results are not shown for the sample without a RIMA, because the position of the rear boundary is not sufficiently well defined, and $K$ can be assumed to be zero.

The $N A$ value corresponding to the applied RIMA is shown along the horizontal axes on the top of each graph. One can see from this figure that the application of RIMA significantly increases the rear boundary contrast. The dependence of the contrast $K$ on the detection angle is non-monotonous, which can be explained by the fact that for each calculation a different model multilayer sample with a random layer boundary shape was generated. Each individual shape determines the characteristics of the sample's OCT image.

From the figures related to the application of isopropanol and 1-pentanol (Figure 7a and 7b), we may conclude that an increase in the detection angle and the coherence length decreases the contrast $K$ non-significantly.

An increase in the detection angle $\eta$ results in a corresponding increase in the contribution of the multiply scattered photons, which introduces distortions and noise into the images and, consequently, decreases the contrast $K$. When the smaller $\eta$ values are used, the photons that exit the paper at angles close to normal to the surface mostly contribute to the signal. On the contrary, when higher $\eta$ values are used, the photons that significantly deflect from the normal contribute to the signal. Small values of $\eta$ provide high photon selectivity and the brightness corresponding to different depths is about the same, while when increasing $\eta$ the selectivity becomes worse and the contribution of multiply scattered photons to the OCT image increases. However, the intensity of the incident beam decreases with depth causing the difference in the brightness, which is not visible for small $\eta$ values due to high selectivity.

The effect of an increase in coherence length on the contrast $K$, on the other hand, is caused by the broadening of the partial signal from individual scatterers, which leads to an overlapping of the partial signals from two neighboring scatter- 


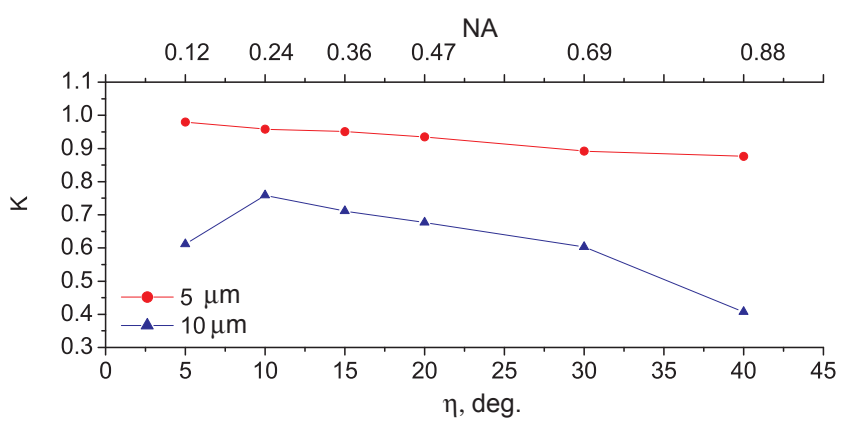

(a)

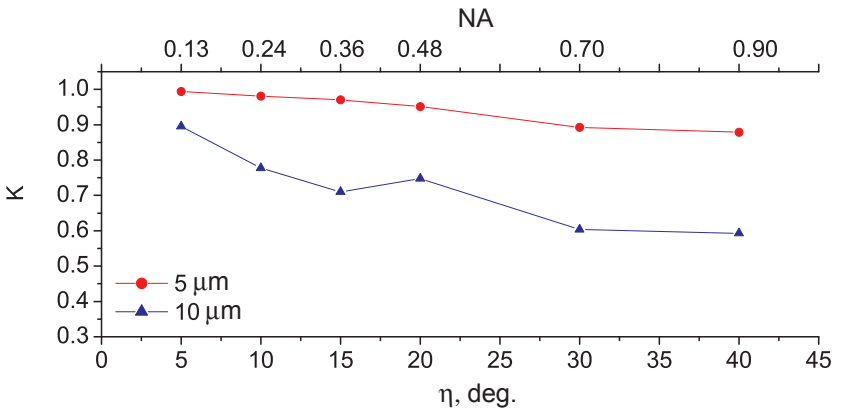

(b)

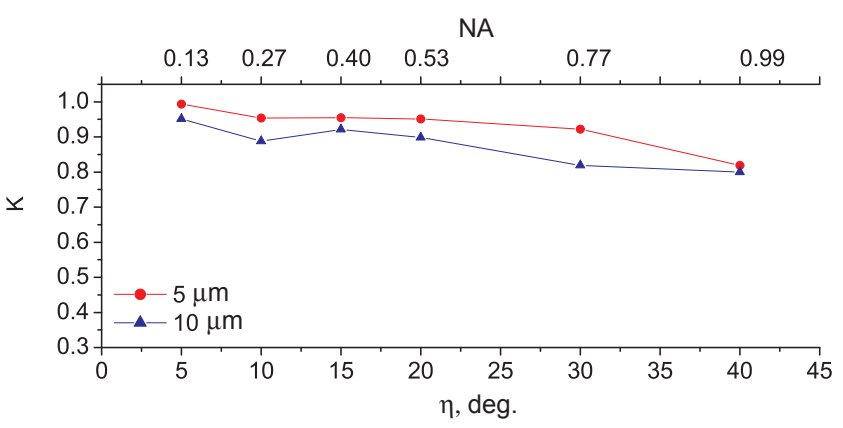

(c)

FIG. 7 Contrast $K$ versus the detection angle $\eta$ (numerical aperture $N A$ ) in the visualization of sample rear boundaries following the application of various RIMAs ( $a$ isopropanol, b - 1-pentanol, c - benzyl alcohol) for two values of the coherence length: $l_{\text {coh }}$ of 5 and $10 \mu \mathrm{m}$

ers, when the distance between them is less than the coherence length. Thus, signals from the neighboring fiber layer boundaries overlap and the signal level corresponding to the air layer between the fiber layers increases. For benzyl alcohol (Figure 7c), the dependence is not monotonous due to the effect of random boundary shape on the OCT image described above and accounts for the rear fiber layer signal level, because the rear boundary cannot be distinguished.

However, we should mention that for all considered values of the coherence length and detection angle, the contrast $K$ is higher than 0.8 for benzyl alcohol, while for other considered RIMAs, it is lower than 0.8 , when $l_{\text {coh }}=10 \mu \mathrm{m}$. For $l_{\text {coh }}=5$ $\mu \mathrm{m}$, the effect of all considered RIMAs is similar. This fact confirms the experimentally based conclusion made in Reference [5] that benzyl alcohol is the best RIMA for paper samples.

\section{CONCLUSIONS}

In this work, the experimental OCT images are obtained with and without application of refractive index matching agents (RIMAs). Speckle suppression is performed by connecting a piezo element with a frequency rate of about $15 \mathrm{~Hz}$ to the scanning head for the spatial averaging of A-scans. This speckle suppression method is shown to be effective for enhancing the obtained OCT images of paper samples.

Multilayer paper model accounting for the non-uniform structure of paper sheets is developed for the calculation of light propagation in paper both before and after the application of RIMAs. The model is applied to simulations of two-dimensional OCT images of paper samples. A qualitative agreement between simulated and experimental images allows concluding that the proposed simple model provides reasonably good results that can be used to analyze the formation of signals in an OCT setup. Potentially it can be used to verify the results obtained with other light scattering measurement techniques. An essential disagreement observed between simulated and experimental images originates from the fact that the structure of real samples is more disordered that that of the simulated samples, where disorder is modeled utilizing a random component in the analytical expression determining the shape of layer boundaries, while the basic layer shape is regular.

However, the effects of the RIMAs employed in the experiment and simulations are similar: they cause a decrease in the contribution of multiply scattered photons, thus reducing the distortions and noise into OCT images, increasing the contrast in the deeper layer images and significantly improving the visualization of each sample's rear boundary.

Variance in the parameters of the simulated OCT setup, coherence length and the detector's numerical aperture shows that an increase in detection angle and coherence length reduces the contrast in the rear boundary of samples. However, the application of RIMAs allows us to detect this border. Benzyl alcohol is shown to be the most effective optical clearing agent among these tested providing the highest rear boundary contrast under the considered conditions.

\section{ACKNOWLEDGEMENTS}

This work was financially supported by the GETA Graduate School and Infotech Oulu. M.Yu. Kirillin acknowledges also the scholarship of Tauno Tönning Foundation. In addition, A.V. Priezzhev acknowledges the scholarship of the Rector of the University of Oulu.

\section{References}

[1] K. Niskanen, Paper Physics, Papermaking Science and Technology, vol. 16 (Fapet Oy, Jyvaskyla, 1998).

[2] J. Borch et al., Handbook of Physical Testing of Paper, vol. 2 (Marcel Dekker, New York, 2002). 
[3] VTT Automation and Helsinki University of Technology, "Knowpap - e-Learning Environment for Papermaking and Automation", http://www.knowpap.com.

[4] B. Bouma and G. Tearney (eds.), Handbook of Optical Coherence Tomography (Marcel Dekker, New York, 2002).

[5] T. Fabritius, E. Alarousu, T. Prykari, J. Hast, and R. Myllyla, “Characterization of optically cleared paper by optical coherence tomography" Quantum Electron. 36, 181-187 (2006).

[6] E. Alarousu, L. Krehut, T. Prykari, and R. Myllyla, "Study on the use of optical coherence tomography in measurements of paper properties" Meas. Sci. Technol. 16, 1131-1137 (2005).

[7] M. Kirillin, A. Priezzhev, J. Hast, and R. Myllyla, "Monte Carlo simulation of optical clearing of paper in optical coherence tomography" Quantum Electron. 36, 174-180 (2006).

[8] K. Green, L. Lamberg, and K. Lumme, "Stochastic modelling of paper structure and Monte Carlo simulation of light scattering" Appl.0ptics 39, 4669-4683 (2000).

[9] M. Mischenko, L.Travis, and A. Lacis, Multiple Scattering of Light by Particles (Cambridge University Press, Cambridge, 2006).
[10] V. Tuchin, Optical Clearing of Tissues and Blood (SPIE Press, Bellingham, Wash., 2006).

[11] V. Tuchin, "Optical clearing of tissues and blood using the immersion method" J. Phys. D: Appl. Phys. 38, 2497-2518 (2005).

[12] K. Bestem'yanov, V. Gordienko, A. Ivanov, A. Konovalov, and A. Podshivalov, "Optical heterodyning study of the propagation dynamics of IR femtosecond laser pulses in a strongly scattering porous medium" Quantum Electron. 34, 666-668 (2004).

[13] J. Saarela and R. Myllyla, "Changes in the time-of-flight of a laser pulse during paper compression" J. Pulp Pap. Sci. 29, 224-227 (2003).

[14] J. Saarela, M. Tormanen, and R. Myllyla, "Measuring pulp consistency and fines content with a streak camera" Meas. Sci. Technol. 14, 1801-1806 (2003).

[15] J. Silvy, "The paper and its unsuspected dimensions", Materials Science Forum 455-456, 781-786 (2004).

[16] J. Carlsson et al., "Time resolved studies of light propagation in paper" Appl.0ptics 34, 1528-1535 (1995). 